Tropical Journal of Pharmaceutical Research March 2019; 18 (3): 619-623

ISSN: 1596-5996 (print); 1596-9827 (electronic)

(C) Pharmacotherapy Group, Faculty of Pharmacy, University of Benin, Benin City, 300001 Nigeria.

\title{
Changes in three tumor markers in patients with advanced lung adenocarcinoma after combination therapy with pemetrexed and platinum: Short-term treatment efficacy
}

\author{
Yang Lou, Bo Xu, Xianshuai Li, Xiaoyi Xu, Xianguo Chen* \\ Cardiothoracic Surgery Department, JinHua Hospital of ZheJiang University, JinHua Municipal Central Hospital, No. 365 \\ Renmin East Road, Jinhua, Zhejiang 321000, China
}

*For correspondence: Email: jz1183@163.com

\begin{abstract}
Purpose: To study changes in three tumor markers before and after combination treatment with pemetrexed and platinum, and evaluate the short-term therapeutic effectiveness of the treatment in advanced lung adenocarcinoma $(A L A)$ subjects.

Method: Patients with ALA $(n=120)$ admitted to JinHua Municipal Central Hospital from January 2017 to May 2018 were selected as the research subjects. According to the results of chemotherapy, they were divided into two groups: significantly-reduced tumor volume group (90 cases), and nonsignificantly-reduced tumor volume group (21 cases). The two groups were treated with chemotherapy using a combination of pemetrexed and cisplatin. The levels of CEA, CA125 and CYFRA21-1 before and after chemotherapy were assayed to determine their relationship with short-term therapy effectiveness.

Result: Overall analysis of tumor markers in the 120 patients with ALA showed statistically significant reduction in overall tumor marker levels before and after chemotherapy $(p<0.05)$. At the end of chemotherapy, the tumor markers were markedly reduced in subjects with significant tumor volume, and there was statistical difference between the two groups before chemotherapy $(p<0.05)$. Changes in CEA, CA125 and CYFRA21-1 were positively correlated with the chemotherapeutic effects on patients with ALA.

Conclusion: In ALA patients treated with pemetrexed and platinum, changes in serum CEA, CA125 and CYFRA21-1 profiles before and after treatment depend on the effect of chemotherapy, and they are reliable for prediction of short-term therapeutic efficacy.
\end{abstract}

Keywords: CEA, CA125, CYFRA21-1, Lung adenocarcinoma, Short-term efficacy

This is an Open Access article that uses a funding model which does not charge readers or their institutions for access and distributed under the terms of the Creative Commons Attribution License (http://creativecommons.org/licenses/by/4.0) and the Budapest Open Access Initiative (http://www.budapestopenaccessinitiative.org/read), which permit unrestricted use, distribution, and reproduction in any medium, provided the original work is properly credited.

Tropical Journal of Pharmaceutical Research is indexed by Science Citation Index (SciSearch), Scopus, International Pharmaceutical Abstract, Chemical Abstracts, Embase, Index Copernicus, EBSCO, African Index Medicus, JournalSeek, Journal Citation Reports/Science Edition, Directory of Open Access Journals (DOAJ), African Journal Online, Bioline International, Open-J-Gate and Pharmacy Abstracts

\section{INTRODUCTION}

Cancer of the lung cancer is the most frequently occurring malignant tumor in the world, and its incidence is rising. According to histopathological classification, lung cancer comprises small cell lung cancer and non-small cell lung cancer. Lung cell adenocarcinoma belongs to the category of non-small cell lung cancer. At present, the combination of platinum and drugs is the first-line 
standard chemotherapy for ALA. Clinically, a variety of tumor markers are associated with lung cancer. These tumor markers reflect the occurrence, classification, therapeutic effects, and prognosis of lung cancer subjects [1]. This study was performed in patients with ALA who received pemetrexed plus cisplatin with the aim of comparing the serum levels of CEA, CA125 and CYFRA21-1. Moreover, the predictive value of these tumor markers was determined, with a view of providing a basis for the treatment of patients with ALA.

\section{METHODS}

\section{Baseline data}

A total of 120 patients with ALA admitted to our hospital from January 2017 to May 2018 were selected as research subjects. They comprised 55 males and 65 females, aged $52-87$ years (mean age $=73.8 \pm 7.97$ years). They were grouped in accordance with RECIST standard [2]. Tumor length reduction of more than $30 \%$ was classified as significantly reduced tumor volume, while tumor length reduction less than $30 \%$ was classified as non-significantly reduced tumor volume. This study received approval from the Ethical Committee of Cardiothoracic Surgery Department, JinHua Hospital of ZheJiang University, JinHua Municipal Central Hospital, No. 365 Renmin East Road, Jinhua, Zhejiang, China (approval no. 201510513). It was carried out in accordance with the guidelines of the Helsinki Declaration of 1964 as amended in 1996 [3].

\section{Treatments}

The general data of patients used in the study were collected and reviewed. On admission, the patients were subjected to routine examinations such as chest CT and enhanced CT to record the tumor sites. Vital signs were monitored, and tests for liver, lung and kidney functions were carried out, in addition to routine blood tests. Detected abnormalities were corrected to enable patients receive chemotherapy. Nutritional support treatment was also given. Vitamin B12 and folic acid were administered for the first 7 days of pemetrexed and cisplatin chemotherapy, and up to 21 days after completion of chemotherapy. Pemetrexed $\left(500 \mathrm{mg} / \mathrm{m}^{2}\right.$, Jiangsu Haosen Pharmaceutical Co. Ltd) was given by intravenous infusion along with cisplatin (75 $\mathrm{mg} / \mathrm{m}^{2}$ ). Cisplatin was applied in the first three days of chemotherapy. Appropriate treatments were given for adverse reactions such as nausea and vomiting. The patients' blood routine, electrolytes, and liver and kidney function were closely monitored so as to avoid serious side effects. Chemotherapy was given in two cycles (1 cycle every 3 weeks).

\section{Biochemical assays}

Fasting blood samples were taken before the end of chemotherapy and at the end of 2 cycles of chemotherapy for assay of tumor markers (CEA, CA125 and CYFRA21-1). The assays were done using enzyme-linked immunosorbent assay (ELISA) kits (Shanghai Lanji Biotechnology Co. Ltd).

\section{Statistical analysis}

The research data were statistically analyzed using SPSS 19.0 software. The measurement data were in accordance with normal distribution and were expressed as mean \pm standard deviation (SD). Comparison between groups was done with Student's $t$ test. Correlation between groups was analyzed by Spearman test. Values of $p<0.05$ were considered statistically significant.

\section{RESULTS}

\section{Patients with different short-term effects}

As depicted in Table 1, there were 99 cases in which the tumor volume was significantly reduced, and 21 cases in the non-significant reduction group. There were no significant differences in clinical profiles of the two groups ( $p$ $>0.05$ ).

Table 1: Clinical outcome in subjects with different short-term efficacy

\begin{tabular}{lcccc}
\hline General information & $\begin{array}{c}\text { Group with significantly } \\
\text { reduced tumor volume }\end{array}$ & $\begin{array}{c}\text { Group in which tumor } \\
\text { volume was non-significantly } \\
\text { reduced }\end{array}$ & $\boldsymbol{t}^{\mathbf{\chi}^{2}}$ & $\boldsymbol{p}$ \\
\hline Age (years) ${ }^{*}$ & $72.6 \pm 6.72$ & $79.1 \pm 5.31$ & 1.836 & 0.069 \\
Gender (male/female) & $48 / 51$ & $7 / 14$ & 1.602 & 0.206 \\
Smoking history (Yes/No) & $49 / 50$ & $11 / 10$ & 0.058 & 0.810 \\
Symptom (Yes/No) & $62 / 37$ & $15 / 6$ & 0.584 & 0.445 \\
Diseased region (left/right) & $40 / 59$ & $9 / 12$ & 0.043 & 0.835 \\
\hline
\end{tabular}


Table 2: Changes in levels of tumor markers in ALA patients before and after chemotherapy (mean $\pm S D$ )

\begin{tabular}{lcccc}
\hline Tumor marker & Before chemotherapy & After chemotherapy & $\boldsymbol{t}$ & $\boldsymbol{p}$ \\
\hline CEA $(\mathrm{ng} / \mathrm{mL})$ & $38.35 \pm 11.05$ & $22.47 \pm 9.58$ & 2.227 & 0.028 \\
CA125 $(\mathrm{U} / \mathrm{mL})$ & $90.17 \pm 2.83$ & $59.25 \pm 17.56$ & 2.178 & 0.031 \\
CYFRA21-1 $(\mathrm{ng} / \mathrm{mL})$ & $18.21 \pm 5.62$ & $10.89 \pm 6.50$ & 2.085 & 0.039 \\
\hline
\end{tabular}

Table 3: Changes in tumor markers before and after chemotherapy in patients with different short-term effects

\begin{tabular}{|c|c|c|c|c|}
\hline \multirow{2}{*}{ Tumor marker } & \multicolumn{2}{|c|}{$\begin{array}{l}\text { Significantly-reduced tumor volume } \\
\text { group }\end{array}$} & \multicolumn{2}{|c|}{$\begin{array}{l}\text { Non-significantly reduced tumor } \\
\text { volume group }\end{array}$} \\
\hline & $\begin{array}{l}\text { Before } \\
\text { chemotherapy }\end{array}$ & $\begin{array}{l}\text { After } \\
\text { chemotherapy }\end{array}$ & $\begin{array}{l}\text { Before } \\
\text { chemotherapy }\end{array}$ & $\begin{array}{l}\text { After } \\
\text { chemotherapy }\end{array}$ \\
\hline $\begin{array}{l}\text { CEA (ng } / \mathrm{mL}) \\
\text { CA125 }(\mathrm{U} / \mathrm{mL}) \\
\text { CYFRA21-1 (ng/mL) }\end{array}$ & $\begin{array}{l}39.28 \pm 7.32^{2} \\
89.89 \pm 5.05^{4} \\
17.99 \pm 2.88^{6}\end{array}$ & $\begin{array}{l}20.30 \pm 6.81^{1} \\
52.85 \pm 6.27^{3} \\
8.51 \pm 5.15^{5}\end{array}$ & $\begin{array}{l}34.14 \pm 5.63^{\mathrm{a}} \\
91.02 \pm 7.52^{\mathrm{b}} \\
18.10 \pm 4.25^{\mathrm{c}}\end{array}$ & $\begin{array}{l}30.26 \pm 9.58^{2 \mathrm{~d}} \\
88.04 \pm 5.13^{4 \mathrm{e}} \\
17.0 \pm 6.27^{6 \mathrm{t}}\end{array}$ \\
\hline $\begin{array}{l}{ }^{1-6} p<0.05\left({ }^{1} t=2.157\right. \\
{ }^{5} p=0.047,{ }^{6} t=2.207 \\
\text { reduced tumor volum } \\
\text { corresponding values } \\
{ }^{c} p=0.061\end{array}$ & $.033,{ }^{2} t=2.024$ & $\begin{array}{r}0.039,{ }^{3} t=2.10 t \\
\text { n levels before c }\end{array}$ & $\begin{array}{l}=0.038,{ }^{4} t=2.1 \\
\text { therapy. In the }\end{array}$ & $\begin{array}{l}0=0.047,{ }^{5} t=2.007, \\
o \text { of non-significantly } \\
\text { omparable with their }\end{array}$ \\
\hline
\end{tabular}

\section{Changes in tumor markers before and after chemotherapy in patients with ALA}

Tumor markers in the 120 ALA patients were determined. As shown in Table 2, the overall tumor marker levels of the patients were significantly decreased before and after chemotherapy $(p<0.05)$.

\section{Changes in tumor markers before and after chemotherapy with different short-term effects}

Table 3 shows that CEA, CA125 and CYFRA211 were higher in the significantly reduced tumor volume and non-significantly reduced tumor volume groups before chemotherapy, but there was no statistical difference between them $(p>$ 0.05). In significantly-reduced tumor volume group, compared with before chemotherapy, the tumor markers were significantly decreased after chemotherapy. There were no significant differences in the levels of the three tumor markers in the non-significantly reduced tumor volume before and after chemotherapy $(p>$ 0.05).

\section{Correlation between changes in tumor markers and chemotherapy effects}

Table 4: Correlation analysis between changes in tumor markers and chemotherapy effects

\begin{tabular}{lcc}
\hline $\begin{array}{l}\text { Tumor } \\
\text { marker }\end{array}$ & $\mathbf{R}$ & $\begin{array}{c}\boldsymbol{P} \text { - } \\
\text { value }\end{array}$ \\
\hline CEA & 0.917 & 0.000 \\
CA125 & 0.835 & 0.000 \\
CYFRA21-1 & 0.798 & 0.000 \\
\hline
\end{tabular}

The results in Table 4 show that changes in CEA, CA125 and CYFRA21-1 were positively correlated with chemotherapy effects in the patients.

\section{DISCUSSION}

Most patients with lung adenocarcinoma show no obvious clinical symptoms in the early stages. Thus, they are already in the advanced stages of III and IV at the time of diagnosis, and so can only be treated with internal medicine such as chemotherapy and radiotherapy [4]. Platinum drugs are used as first-line chemotherapy for lung malignant tumors, and often need to be combined with other chemotherapy drugs to form a first-line chemotherapy program.

Pemetrexed is a new class of cytotoxic and antimetabolic chemotherapeutic drugs that kill tumor cells by interfering with folate biosynthesis. Pemetrexed has demonstrated definitive clinical effects in the treatment of lung adenocarcinoma, even as researchers believe it can be used as a primary chemotherapy for non-small cell pulmonary carcinoma. It has been reported that pemetrexed and gemcitabine combined with platinum drugs have the same level of therapeutic effects, but the former is less toxic, and is associated with greater patient tolerance [5].

Other studies have also suggested that pemetrexed has the advantage of being less toxic [6]. Needless to say, the chemotherapeutic effect in subjects with advanced pulmonary cancer is exact. However, in clinical evaluation, in addition to imaging, there are no reliable, intuitive and simple methods for evaluating chemotherapy results. Another difficult problem 
involves how to monitor the patient's condition during the chemotherapy cycle so as to adjust the treatment plan in time.

Tumor markers are activated by protooncogenes and tumor suppressor genes. They can be used to predict therapeutic effects, to monitor whether tumors recur, to judge the prognosis of tumor patients, to detect metastatic tumors of unknown origin, and to detect primary tumors [7-9]. The present research has confirmed that changes in tumor markers correspond with the progression or degree of malignancy of malignant tumors. The use of tumor markers as effective indicators for disease monitoring and prognosis evaluation is the main trend in malignant tumor-related evaluation research. Studies [10] have shown that increased levels of the tumor markers CEA, CA125 and CYFRA21-1 in lung cancer subjects reflect the effects of chemotherapy. This is also the reason why the present study selected these three kinds of tumor markers in many lung malignant tumor-related tumors.

The tumor marker CEA is highly abundant in cancers derived from endodermal differentiation such as digestive tract adenocarcinoma and lung adenocarcinoma, and is currently the most commonly used screening marker for malignant tumors in clinical practice. Research on lung carcinoma has demonstrated that CEA levels can effectively predict patients' prognosis and response to related treatments. Elevated CEA profiles are associated with significantly shorter mean survival after surgery, radiotherapy and chemotherapy than lower CEA levels. Thus, CEA can be used as a predictor of prognosis in patients with malignant lung tumors [11].

The carbohydrate antigen CA125 is highly expressed in female reproductive system malignancies, and its serum levels in lung cancer patients have also been abnormally elevated in recent years. This phenomenon has made it a new focus in the study of malignant tumors in the lungs. The tumor marker CYFRA21-1 is new, and it has been widely studied in recent years. It is a soluble fragment of keratin intermediate filament 19 in cells, and is important for the diagnosis and monitoring of lung cancer [12]. The diagnosis and evaluation related to malignant tumors have high research prospects. In the present study, serum profiles of CEA, CA125 and CYFRA21-1 decreased significantly at the end of chemotherapy.

Further analysis of the changes in the three tumor markers in patients with different shortterm effects revealed that the effect was significant: the patients with significantly reduced tumor volume had decreased CEA, CA125, CYFRA21-1 levels, while their profiles did not change in subjects treated ineffectively. This trend of change is consistent with the trend of tumor volume in patient imaging. It indicates that the changes in the levels of these three tumor markers in the tissue fluid and serum of patients can assist in the diagnosis of lung cancer, reflecting the effect of treatment of advanced lung adenocarcinoma [13-16].

It can be speculated that if the profiles of these tumor markers are significantly increased, they either indicate that the patients with lung adenocarcinoma are in advanced stage, or that long-term treatment is not satisfactory. In addition, if the patient's treatment effect is satisfactory, the serum profiles levels of CEA, CA125 and CYFRA21-1 may be significantly lowered. Similarly, if their profiles are basically unchanged or slightly reduced, they suggest that the effect of chemotherapy on lung adenocarcinoma did not reach the expected outcome, or that the patient's condition is even worsened.

Preliminary analysis of tumor markers can be used to assess the effect of treatment because the tumor markers are synthesized in tumor cells. When the chemotherapy effect is good and can kill most lung adenocarcinoma tumor cells, the tumor markers released into the body fluids are synthesized. When the chemotherapy drugs are reduced, the levels of tumor markers are also reduced. However, tumor cells cannot be eliminated by the chemotherapeutic drugs alone. Indeed, the degree of elimination may not achieve the expected effect, and the tumor cells may even continue to increase, so that the content of tumor markers released by their biosynthesis is not reduced. Being significantly increased, there is clinically significant fluctuation in the profiles of CEA, CA125 and CYFRA21-1.

\section{Limitations of the study}

Few participants were included in this study. In addition, the study was based on one single center. Therefore, the application of the conclusion drawn from this work should be done with caution.

\section{CONCLUSION}

In the use of pemetrexed-platinum co-treatment for advanced pulmonary adenocarcinoma, changes in serum profiles of the three tumor markers CEA, CA125, and CYFRA21-1 before and after treatment depend on the effect of 
chemotherapy. These results are considered beneficial in the assessment of short-term effects.

\section{DECLARATIONS}

\section{Conflict of Interest}

No conflict of interest associated with this work.

\section{Contribution of Authors}

We declare that this work was done by the author(s) listed in this manuscript. All liabilities arising from claims relating to the manuscript content will be borne by the authors. All authors read and gave approval for publication of the manuscript. Xianguo Chen conceived and designed the study. Yang Lou, Xianguo Chen, Bo Xu, Xianshuai Li and Xiaoyi Xu collected and analyzed the data, while Yang Lou wrote the manuscript.

\section{REFERENCES}

1. Du ZY. The Diagnostic Value of Tumor Markers CEA, CYFRA21-1 and TPS Joint Detection in Senile Nonsmall Cell Lung Cancer. Med Innov China 2017; 14(30): 4-7.

2. Ko SF, Yip HK, Lee CC, Su $\mathrm{CH}$, Huang $\mathrm{CC}, \mathrm{Ng} \mathrm{SH}$, Chen YL, Chen MC. Apparent Diffusion Coefficient is a Useful Biomarker for Monitoring Adipose-Derived Mesenchymal Stem Cell Therapy of Renal IschemicReperfusion Injury. Mol Imaging Biol 2018; 20(5): 750 760.

3. World Health Organization. Declaration of Helsinki. $\mathrm{Br}$ Med J 1996; 313(7070): 1448-1449.

4. Ministry of Health of the People's Republic of China. The main situation of the third national cause of death investigation. Chin J Oncol 2008; 17(5): 344-345.

5. Su S, Lü JQ, Li N, Zhang XL. Clinical efficacy of pemetrexed and gemcitabine combined with platinum first-line treatment of advanced lung adenocarcinoma. $J$ Pract Med 2011; 27(8): 1458-1460.
6. Wang Wei. Therapeutic effect of RhG-CSF on leukopenia after chemotherapy in advanced lung cancer. Pract Med Clin 2010; 13(1): 71-72.

7. Zhang just. Effect of Fuzheng Kangai Recipe Combined with Chemotherapy on Serum Tumor Markers in Patients with Advanced Non-small Cell Lung Cancer. Shanxi Tradit Chin Med 2017; 38(7): 885-886.

8. Wang Y, Fan HS, Zhang W, Li XQ. Correlation between serum tumor markers in advanced non-small cell lung cancer and objective efficacy and survival time of second-line chemotherapy. Chin J Med 2017; 29(10): 21-25.

9. Ji DC. The value of serum tumor marker level in evaluating the therapeutic effect of advanced non-small cell lung cancer. Chin Med 2016; 11(2): 199-202.

10. Shi $W$, Wang $L$. The relationship between the main tumor markers of lung adenocarcinoma and chemotherapy efficacy. J Pract Oncol 2015; 30(4): 377-378.

11. Tax LL. Clinical significance of detection of CEA, CYFRA21-1, NSE and SCC in serum of patients with lung cancer. J Xi'an Jiaotong Univ: Med Sci 2010; 31(6): 708-710.

12. Wang $L$, Jiang $X D$, Chen $Y$, Liu YR, Liu PK, Xu L. Targeted therapeutic effect and serum tumor marker changes in patients with advanced non-small cell lung cancer. Cancer Progress 2017; 15(9): 1069-1072.

13. Wang WT, Zhang GJ. The value of combined detection of CEA, CYFRA21-1, NSE and CA125 in the diagnosis of lung cancer. Chin Lab Diagn 2014; 2: 224-226.

14. $\mathrm{Yu} \mathrm{ZH}$, Meng W. Evaluation of the diagnostic value of four tumor markers in serum for lung cancer. Labeled Immunoassays Clin Med 2016; 6: 655-658.

15. Mashaly AH, Anwar R, Ebrahim MA, Eissa LA, MS El. Diagnostic and Prognostic Value of Talin-1 and Midkine as Tumor Markers in Hepatocellular Carcinoma in Egyptian Patients. Asian Pac J Cancer Prev 2018; 19(6):1503-1508.

16. Lu B, Wu HK, Yao FF, Zhang CC. Comparison of the efficacy of gemcitabine combined with pemetrexed in combination with cisplatin in the treatment of non-small cell lung cancer and its effect on serum tumor markers. J Pract Cancer 2017; 32(6): 975-977. 